\title{
On the Approximate Calculation of Double Integrals
}

\author{
By Moshe Levin
}

\begin{abstract}
Cubature formulas are obtained which are optimal or asymptotically optimal on given sets of functions. These formulas consist of line integrals which may be evaluated by optimal or asymptotically optimal quadrature formulas. The advantage of these formulas over the optimal and asymptotically optimal cubature formulas with rectangular-lattices of knots is shown.
\end{abstract}

1. Introduction. Notations and Definitions. The problem of constructing efficient cubature formulas is a difficult problem. The results in this field are reviewed in [1]-[4]. Below we consider the problem of obtaining optimal and near to optimal cubature formulas. The approach is based on the idea of blending [5], [6].

Let $1<p \leqslant \infty, r, s \geqslant 1, M, N, P$ be given, $p^{-1}+q^{-1}=1, D=[0,1] \times[0,1]$. Let us introduce the notation of derivatives

$$
f^{(i, j)}(x, y)=\frac{\partial^{i+j}}{\partial x^{i} \partial y^{j}} f(x, y), \quad i, j=0,1, \ldots,
$$

of norms

$$
\begin{aligned}
\|f(\cdot)\|_{p} & = \begin{cases}\left(\int_{0}^{1}|f(x)|^{p} d x\right)^{1 / p}, & p<\infty, \\
\operatorname{supvrai}_{0 \leqslant x \leqslant 1}|f(x)|, & p=\infty,\end{cases} \\
\|f(\cdot, \cdot)\|_{p} & = \begin{cases}\left(\iint_{D}|f(x, y)|^{p} d x d y\right)^{1 / p}, & p<\infty, \\
\operatorname{supvrai}_{(x, y) \in D}|f(x, y)|, & p=\infty,\end{cases}
\end{aligned}
$$

and the sets of functions

$$
\begin{gathered}
W_{p}^{\mu}=\left\{f(x): f^{(\mu)}(x) \text { piecewise continuous on }[0,1],\left\|f^{(\mu)}(\cdot)\right\|_{p} \leqslant 1\right\}, \\
V_{p}^{r, s}=\left\{f(x, y): f^{(i, j)}(x, y)(i \leqslant r, j \leqslant s) \text { piecewise continuous on } D,\right. \\
\left.\left\|f^{(r, s)}(\cdot, \cdot)\right\|_{p} \leqslant M\right\}, \\
U_{p}^{r, s}=\left\{f(x, y): f \in V_{p}^{r, s},\left\|f^{(r, 0)}(\cdot, y)\right\|_{p} \leqslant N, \forall y \in[0,1],\right. \\
\left.\left\|f^{(0, s)}(x, \cdot)\right\|_{p} \leqslant P, \forall x \in[0,1]\right\},
\end{gathered}
$$

Received January 13, 1982; revised June 21, 1982.

1980 Mathematics Subject Classification. Primary 65D30; Secondary 41A55. 


$$
\begin{gathered}
\tilde{V}_{p}^{r, s}=\left\{f(x, y): f \in V_{p}^{r, s}, f^{(i, 0)}(0, y) \equiv f^{(i, 0)}(1, y), i=0, \ldots, r-1,\right. \\
\left.f^{(0, j)}(x, 0) \equiv f^{(0, j)}(x, 1), j=0, \ldots, s-1\right\}, \\
\tilde{U}_{p}^{r, s}=\left\{f(x, y): f \in U_{p}^{r, s} \cap \tilde{V}_{p}^{r, s}\right\}, \\
W_{p}^{r, s}=\left\{f(x, y): f \in V_{p}^{r, s},\left\|\int_{0}^{1} f^{(r, 0)}(\cdot, y) d y\right\|_{p} \leqslant N,\left\|\int_{0}^{1} f^{(0, s)}(x, \cdot) d x\right\|_{p} \leqslant P\right\} .
\end{gathered}
$$

Let $Q$ be a set of linear functionals defined on a set $H$ of functions $f$, and let $L$ be a given linear functional defined on the set $H$. Consider approximate formulas of the form

$$
L f=\sum_{k=1}^{l} L_{k} f+r(f), \quad L_{k} \in Q, k=1, \cdots l,
$$

where $r(f)$ is the error of the formula.

Formula (1.1) is called an optimal formula on the set $H$, if the functionals $L_{k} \in Q$ are chosen so that the quantity

$$
r[H]=\sup _{f \in H}|r(f)|
$$

has the minimal value.

Denote the minimal value of $r[H]$ by $r^{*}[H]$.

Formula (1.1) is called asymptotically optimal on the set $H$, if the functionals $L_{K} \in Q$ are chosen so that

$$
\lim _{l \rightarrow \infty} \frac{r^{*}[H]}{r[H]}=1
$$

Designate

$$
\begin{aligned}
& A_{1}^{(l)}, \ldots, A_{l}^{(l)} ; x_{1}^{(l)}, \ldots, x_{l}^{(l)} ; r_{1}^{(l)}, \\
& B_{1}^{(l)}, \ldots, B_{l}^{(l)} ; y_{1}^{(l)}, \ldots, y_{l}^{(l)} ; r_{2}^{(l)},
\end{aligned}
$$

the coefficients, knots and the value (1.2) of the optimal formulas

$$
\int_{0}^{1} f(x) d x=\sum_{k=1}^{l} A_{k} f\left(x_{k}\right)+r(f), \quad 0 \leqslant x_{1}<\cdots<x_{l} \leqslant 1,
$$

on the sets $W_{p}^{r}$ and $W_{p}^{s}$, respectively.

It follows by the results of [3, Theorem 5.2.1 and 3.6.1] that $r_{1}^{(l)}=O\left(l^{-r}\right)$, $r_{2}^{(l)}=O\left(l^{-s}\right)$. J. Girshovich [3] has obtained an asymptotically optical formula (1.3) on the set $W_{p}^{\mu}$ with the knots $x_{k}=(k-1) /(l-1), k=1, \ldots, l$, and given a simple scheme for the evaluation of the coefficients of this formula.

Designate by

$$
\bar{A}_{1}^{(l)}, \ldots, \bar{A}_{l}^{(l)} ; \bar{r}_{1}^{(l)}, \quad \bar{B}_{1}^{(l)}, \ldots, \bar{B}_{l}^{(l)} ; \bar{r}_{2}^{(l)}
$$

the coefficients and the value (1.2) of the asymptotically optimal formulas (1.3) on the sets $W_{p}^{r}$ and $W_{p}^{s}$, respectively. 
It is shown in [3], [7] that the optimal formula

$$
\begin{aligned}
\iint_{D} f(x, y) d x d y=\sum_{k=1}^{l} \sum_{j=1}^{\nu} C_{k j} f\left(x_{k}, y_{j}\right)+R(f), \\
0 \leqslant x_{1}<\cdots<x_{l} \leqslant 1,0 \leqslant y_{1}<\cdots<y_{\nu} \leqslant 1,
\end{aligned}
$$

on the set $W_{p}^{r, s}$ has coefficients $C_{k j}=A_{k}^{(l)} B_{j}^{(\nu)}$, knots $\left(x_{k}, y_{j}\right)=\left(x_{k}^{(l)}, y_{j}^{(\nu)}\right)$ and exact bound for the remainder

$$
R\left[W_{p}^{r, s}\right]=\sup _{f \in W_{p}^{r, s}}|R(f)|=O\left(l^{-r}+\nu^{-s}\right) .
$$

The asymptotically optical formula (1.5) on the set $W_{p}^{r, s}$ has coefficients $C_{k j}=$ $\bar{A}_{k}^{(l)} \bar{B}_{j}^{(\nu)}$, knots $\left(x_{k}, y_{j}\right)=((k-1) /(l-1),(j-1) /(\nu-1))$ and exact bound for the remainder $O\left(l^{-r}+\nu^{-s}\right)$ [3]. The optimal and asymptotically optimal formulas (1.5) on the set $V_{p}^{r, s}$ do not exist since by Lemma 1.5.1 of [3,pp. 18-19], these formulas must be exact for all functions $\varphi(x) y^{j}, \psi(y) x^{i} \in V_{p}^{r, s}(i<r, j<s)$. Also, we do not know the optimal and asymptotically optimal formulas (1.5) on the set $U_{p}^{r, s}$.

The first optimal quadrature formulas were found by A. Sard in the case of fixed knots [8], by S. Nikolsky [9] and by I. Schoenberg [10]. The first optimal cubature formula with arbitrary knots was presented in [11].

2. Cubature Formulas Based on Optimal Quadrature Formulas. We shall find an optimal formula of the form

$$
\begin{aligned}
\iint_{D} f(x, y) d x d y= & \sum_{k=1}^{m} \alpha_{k} \int_{0}^{1} \alpha_{k} \int_{0}^{1} f\left(x_{k}, y\right) d y+\sum_{j=1}^{n} \beta_{j} \int_{-0}^{1} f\left(x, y_{j}\right) d x \\
& +\sum_{k=1}^{m} \sum_{j=1}^{n} \gamma_{k j} f\left(x_{k}, y_{j}\right)+E(f), \\
& \quad 0 \leqslant x_{1}<\cdots<x_{m} \leqslant 1,0 \leqslant y_{1}<\cdots<y_{n} \leqslant 1,
\end{aligned}
$$

on the set $V_{p}^{r, s}$. In other words, we shall find the formula (2.1) with the minimal value of

$$
E\left[V_{p}^{r, s}\right]=\sup _{f \in V_{p}^{r, s}}|E(f)|
$$

THEOREM 2.1. The coefficients and knots

$$
\begin{array}{ll}
\alpha_{k}=A_{k}^{(m)}, & \beta_{j}=B_{j}^{(n)}, \quad \gamma_{k j}=-A_{k}^{(m)} B_{j}^{(n)}, \\
x_{k}=x_{k}^{(m)}, & y_{j}=y_{j}^{(n)}, \quad k=1, \ldots, m ; j=1, \ldots, n,
\end{array}
$$

and the exact bound of the remainder

$$
E\left[V_{p}^{r, s}\right]=M r_{1}^{(m)} r_{2}^{(n)}
$$

are the coefficients, knots and exact bound of the remainder of the optimal formula (2.1) on the set $V_{p}^{r, s}$.

Proof. The optimal formula (2.1) must satisfy the condition

$$
\begin{aligned}
E\left(\varphi(x) y^{\nu}\right)=E\left(\varphi(y) x^{\lambda}\right)=0 \quad \forall \varphi(x) y^{\nu}, \varphi(y) x^{\lambda} \in V_{p}^{r, s} \\
\nu=0, \ldots, s-1 ; \lambda=0, \ldots, r-1,
\end{aligned}
$$


by Lemma 1.5.1 of [3]. In view of this we only consider formulas (2.1) with the property (2.4). Taking in (2.1) $f(x, y)=\varphi(x) y^{\nu}$ and $f(x, y)=\varphi(y) x^{\lambda}$, where $\varphi(x)$ is an arbitrary function, we obtain by $(2.4)$

$$
\begin{gathered}
\sum_{j=1}^{n} \beta_{j} y_{j}^{\nu}=\frac{1}{\nu+1}, \quad \sum_{k=1}^{m} \alpha_{k} x_{k}^{\lambda}=\frac{1}{\lambda+1}, \\
\frac{\alpha_{k}}{\nu+1}=-\sum_{j=1}^{n} \gamma_{k j} y_{j}^{\nu}, \quad \frac{\beta_{j}}{\lambda+1}=-\sum_{k=1}^{m} \gamma_{k j} x_{k}^{\lambda}, \quad k=1, \ldots, m ; \\
j=1, \ldots, n ; \lambda=0, \ldots, r-1 ; \nu=0, \ldots, s-1 .
\end{gathered}
$$

Now let $f(x, y) \in V_{p}^{r, s}$,

$$
U_{+}^{k}= \begin{cases}U^{k}, & U>0 \\ 0, & U \leqslant 0\end{cases}
$$

By Taylor's formula

$$
\begin{aligned}
f(x, y)= & \sum_{i=0}^{r-1} \psi_{i}(y) x^{i}+\sum_{j=0}^{s-1} \varphi_{j}(x) y^{j} \\
& +\frac{1}{(r-1) !(s-1) !} \iint_{D} f^{(r, s)}(t, u)(x-t)_{+}^{r-1}(y-u)_{+}^{s-1} d t d u,
\end{aligned}
$$

where

$$
\begin{gathered}
\psi_{i}(y)=\frac{1}{i !} f^{(i, 0)}(0, y), \\
\varphi_{j}(x)=\frac{1}{j !(r-1) !} \int_{0}^{1} f^{(r, j)}(t, 0)(x-t)_{+}^{r-1} d t .
\end{gathered}
$$

Hence by (2.4)

$$
\begin{aligned}
E(f) & =\frac{1}{(r-1) !(s-1) !} \iint_{D} f^{(r, s)}(t, u) E\left((x-t)_{+}^{r-1}(y-u)_{+}^{s-1}\right) d t d u \\
& =\iint_{D} f^{(r, s)}(t, u) K(t, u) d t d u
\end{aligned}
$$

where $K(t, u)$ is monospline,

$$
\begin{gathered}
K(t, u)=\frac{1}{(r-1) !(s-1) !}\left[\frac{(1-t)^{r}(1-u)^{s}}{r s}-\frac{(1-u)^{s}}{s} \sum_{k=1}^{m} \alpha_{k}\left(x_{k}-t\right)_{+}^{r-1}\right. \\
-\frac{(1-t)^{r}}{r} \sum_{j=1}^{n} \beta_{j}\left(y_{j}-u\right)_{+}^{s-1} \\
\left.\quad-\sum_{k=1}^{m} \sum_{j=1}^{n} \gamma_{k j}\left(x_{k}-t\right)_{+}^{r-1}\left(y_{j}-u\right)_{+}^{s-1}\right] .
\end{gathered}
$$

The monospline $K(t, u)$ satisfies the condition

$$
K^{(\lambda, 0)}(0, y) \equiv K^{(0, \nu)}(x, 0) \equiv 0, \quad \lambda=0, \ldots, r-1 ; \nu=0, \ldots, s-1,
$$

by the equalities (2.5). 
By Hölder's inequality we obtain from (2.6) that

$$
|E(f)| \leqslant M\|K(\cdot, \cdot)\|_{q} .
$$

For the function

$f_{0}(x, y)=\frac{M}{\|K(\cdot, \cdot)\|_{q}^{q / p}} \iint_{D}|K(t, u)|^{q-1} \operatorname{sgn} K(t, u) \frac{(x-t)_{+}^{r-1}(y-u)_{+}^{s-1}}{(r-1) !(s-1) !} d t d u$ belonging to $V_{p}^{r, s}$, it follows from (2.6) that

$$
E\left(f_{0}\right)=M\|K(\cdot, \cdot)\|_{q} .
$$

Then we have from (2.8) the equality

$$
E\left[V_{p}^{r, s}\right]=M\|K(\cdot, \cdot)\|_{q} .
$$

Now we have to minimize this value.

Consider only functions $K(x, y)$ satisfying condition (2.7) and consider the monosplines

$$
\begin{aligned}
& K_{1}(t)=\frac{(1-t)^{r}}{r !}-\frac{1}{(r-1) !} \sum_{k=1}^{m} \alpha_{k}\left(x_{k}-t\right)_{+}^{r-1}, \\
& K_{1}^{(i)}(0)=0, \quad i=0, \ldots, r-1, \\
& K_{2}(u)= \frac{(1-u)^{s}}{s !}-\frac{1}{(s-1) !} \sum_{j=1}^{n} \beta_{j}\left(y_{j}-u\right)_{+}^{s-1} \\
& K_{2}^{(l)}(0)=0, \quad l=0, \ldots, s-1 .
\end{aligned}
$$

Let

$$
\begin{gathered}
\inf _{\left\{\alpha_{k}, \beta_{j}, \gamma_{k}, x_{k}, y_{j}\right\}}\|K(\cdot, \cdot)\|_{q}=\left\|K^{*}(\cdot, \cdot)\right\|_{q}, \\
\inf _{\left\{\alpha_{k}, x_{k}\right\}}\left\|K_{1}(\cdot)\right\|_{q}=\left\|K_{1}^{*}(\cdot)\right\|_{q}, \quad \inf _{\left\{\beta_{j}, y_{j}\right\}}\left\|K_{2}(\cdot)\right\|_{q}=\left\|K_{2}^{*}(\cdot)\right\|_{q} .
\end{gathered}
$$

It is shown in [3] that

$$
\begin{gathered}
K_{1}^{*}(t)=\frac{(1-t)^{r}}{r !}-\frac{1}{(r-1) !} \sum_{k=1}^{m} A_{k}^{(m)}\left(x_{k}^{(m)}-t\right)_{+}^{r-1}, \\
K_{2}^{*}(u)=\frac{(1-u)^{s}}{s !}-\frac{1}{(s-1) !} \sum_{j=1}^{n} B_{j}^{(n)}\left(y_{j}^{(n)}-u\right)_{+}^{s-1}, \\
\left\|K_{1}^{*}(\cdot)\right\|_{q}=r_{1}^{(m)}, \quad\left\|K_{2}^{*}(\cdot)\right\|_{q}=r_{2}^{(n)} .
\end{gathered}
$$

Also we have the equality

$$
K^{*}(t, u)=K_{1}^{*}(t) K_{2}^{*}(u)
$$

by the result on polynomials of least derivation from zero [7]. This, together with (2.12), give us the values (2.2) and (2.3). The formula (2.1) with coefficients and knots (2.2) satisfies the condition (2.4).

The theorem is proved. 
COROllaRY. Consider the formula

$$
\begin{aligned}
\iint_{D} f(x, y) d x d y= & \sum_{k=1}^{m} \sum_{j=1}^{n^{2}} A_{k}^{(m)} B_{j}^{\left(n^{2}\right)} f\left(x_{k}^{(m)}, y_{j}^{\left(n^{2}\right)}\right) \\
& +\sum_{k=1}^{m^{2}} \sum_{j=1}^{n} A_{k}^{\left(m^{2}\right)} B_{j}^{(n)} f\left(x_{k}^{\left(m^{2}\right)}, y_{j}^{(n)}\right) \\
& -\sum_{k=1}^{m} \sum_{j=1}^{n} A_{k}^{(m)} B_{j}^{(n)} f\left(x_{k}^{(m)}, y_{j}^{(n)}\right)+E_{1}(f) .
\end{aligned}
$$

Let $f(x, y) \in U_{p}^{r, s}$. Then

$$
\left|E_{1}(f)\right| \leqslant N r_{1}^{\left(m^{2}\right)}+P r_{2}^{\left(n^{2}\right)}+M r_{1}^{(m)} r_{2}^{(n)} \text {. }
$$

Proof. We can consider the formula (2.1) with coefficients and knots (2.2) on the set $U_{p}^{r, s}$ as $U_{p}^{r, s} \subset V_{p}^{r, s}$.

Applying optimal quadrature formulas [3]

$$
\begin{aligned}
& \int_{0}^{1} f\left(x, y_{j}^{(n)}\right) d x=\sum_{k=1}^{m^{2}} A_{k}^{\left(m^{2}\right)} f\left(x_{k}^{\left(m^{2}\right)}, y_{j}^{(n)}\right)+r_{1}(f), \\
& \qquad r_{1}(f) \mid \leqslant N r_{1}^{\left(m^{2}\right)}, N^{-1} f(x, y) \in W_{p}^{r} \quad \forall y \in[0,1], \\
& \int_{0}^{1} f\left(x_{k}^{(m)}, y\right) d y=\sum_{j=1}^{n^{2}} B_{j}^{\left(n^{2}\right)} f\left(x_{k}^{(m)}, y_{j}^{\left(n^{2}\right)}\right)+r_{2}(f), \\
& \left|r_{2}(f)\right| \leqslant \operatorname{Pr}_{2}^{\left(n^{2}\right)}, P^{-1} f(x, y) \in W_{p}^{s} \quad \forall x \in[0,1],
\end{aligned}
$$

to the integrals in formula (2.1) with coefficients and knots (2.2), we obtain formula (2.14) and the estimate

$$
\left|E_{1}(f)\right| \leqslant \operatorname{Pr}_{2}^{\left(n^{2}\right)} \sum_{k=1}^{m}\left|A_{k}^{(m)}\right|+N r_{1}^{\left(m^{2}\right)} \sum_{j=1}^{n}\left|B_{j}^{(n)}\right|+M r_{1}^{(m)} r_{2}^{(n)} .
$$

Since by [12] $A_{k}^{(m)}>0, B_{j}^{(n)}>0, k=1, \ldots, m ; j=1, \ldots, n ; A_{1}^{(m)}+\cdots+A_{m}^{(m)}=$ $B_{1}^{(n)}+\cdots+B_{n}^{(n)}=1,(2.15)$ follows.

The corollary is proved.

THEOREM 2.2. The formula

$$
\begin{aligned}
\iint_{D} f(x, y) d x d y= & \frac{1}{m} \sum_{k=1}^{m} \int_{0}^{1} f\left(\frac{k}{m}, y\right) d y+\frac{1}{n} \sum_{j=1}^{n} \int_{0}^{1} f\left(x, \frac{j}{n}\right) d x \\
& -\frac{1}{m n} \sum_{k=1}^{m} \sum_{j=1}^{n} f\left(\frac{k}{m}, \frac{j}{n}\right)+E(f)
\end{aligned}
$$

is the optimal formula (2.1) on the set $\tilde{V}_{p}^{r, s}$. The exact bound for the remainder of this formula is

$$
E\left[\tilde{V}_{p}^{r, s}\right]=\frac{M}{m^{r} n^{s}} B_{r, q} B_{s, q}
$$

where $B_{\mu, q}=\min _{c}\left\|B_{\mu}(\cdot)-c\right\|_{q}$, and $B_{\mu}(x)$ is the Bernoulli polynomial of degree $\mu$. 
The proof is similar to the proof of Theorem 2.1 and uses the optimal quadrature formulas for periodic functions [3] and the formula

$$
f(x, y)=\varphi(x)+\psi(y)+\frac{1}{r ! s !} \iint_{D} f^{(r, s)}(t, u) B_{r}^{*}(x-t) B_{s}^{*}(y-u) d t d u,
$$

where

$$
\begin{gathered}
\varphi(x)=-\frac{1}{r !} \iint_{D} f^{(r, 0)}(t, u) B_{r}^{*}(x-t) d t d u, \quad \psi(y)=\int_{0}^{1} f(t, y) d t, \\
B_{\mu}^{*}(x) \equiv B_{\mu}(x) \quad \text { if } x \in[0,1), \quad B_{\mu}^{*}(x+1) \equiv B_{\mu}^{*}(x) .
\end{gathered}
$$

The latter can be obtained by the analogous one-dimensional formula [13].

Corollary. Consider the formula

$$
\begin{aligned}
\iint_{D} f(x, y) d x d y= & \frac{1}{m n^{2}} \sum_{k=1}^{m} \sum_{j=1}^{n^{2}} f\left(\frac{k}{m}, \frac{j}{n^{2}}\right)+\frac{1}{m^{2} n} \sum_{k=1}^{m^{2}} \sum_{j=1}^{n} f\left(\frac{k}{m^{2}}, \frac{j}{n}\right) \\
& -\frac{1}{m n} \sum_{k=1}^{m} \sum_{j=1}^{n} f\left(\frac{k}{m}, \frac{j}{n}\right)+E_{1}(f) .
\end{aligned}
$$

Let $f(x, y) \in \tilde{U}_{p}^{r, s}$. Then

$$
\left|E_{1}(f)\right| \leqslant \frac{N}{r ! m^{2 r}} B_{r, q}+\frac{P}{s ! n^{2 s}} B_{s, q}+\frac{M}{r ! s ! m^{r} n^{s}} B_{r, q} B_{s, q} .
$$

The proof is similar to the proof of the corollary of Theorem 2.1.

3. Cubature Formulas Based on Asymptotically Optimal Quadrature Formulas. The optimal formula (2.1) and formula (2.14) are based on the optimal quadrature formulas (1.3) on $W_{p}^{\mu}$, which are known only for few values of $\mu$ [3], [4]. Therefore, it is useful to obtain formulas (2.1) and formulas of type (2.14) based on asymptotically optimal quadratures on $W_{p}^{\mu}$, which are known for all integers $\mu[3$, p. 108].

THEOREM 3.1. The coefficients and knots

$$
\begin{aligned}
\alpha_{k}=\bar{A}_{k}^{(m)}, \quad \beta_{j}= & \bar{B}_{j}^{(n)}, \quad \gamma_{k j}=-\bar{A}_{k}^{(m)} \bar{B}_{j}^{(n)}, \\
& x_{k}=\frac{k-1}{m-1}, \quad y_{j}=\frac{j-1}{n-1}, \quad k=1, \ldots, m ; j=1, \ldots, n,
\end{aligned}
$$

and the exact bound for the remainder

$$
E\left[V_{p}^{r, s}\right]=\frac{M B_{r, q} B_{s, q}}{r ! s ! m^{r} n^{s}}\left(1+\frac{C_{1}}{m}\right)^{1 / q}\left(1+\frac{C_{2}}{n}\right)^{1 / q}
$$

are the coefficients, knots and exact bound for the remainder of the asymptotically optimal formula (2.1) on the set $V_{p}^{r, s}$.

The $\bar{A}_{k}^{(m)}, \bar{B}_{j}^{(n)}$ are those alluded to in Section 1, the numbers $B_{\mu, q}$ are determined in Theorem 2.1, and $C_{1}$ and $C_{2}$ are constants independent of $m$ and $n$.

Proof. Consider formula (2.1) on the set $V_{p}^{r, s}$. By (2.9) and (2.13) we have the exact bound for the remainder for the optimal formula (2.1) on the set $V_{p}^{r, s}$.

$$
E^{*}\left[V_{p}^{r, s}\right]=M\left\|K_{1}^{*}(\cdot)\right\|_{q}\left\|K_{2}^{*}(\cdot)\right\|_{q} .
$$


Consider formula (2.1) with the corresponding monospline

$$
K(t, u)=\bar{K}(t, u)=\bar{K}_{1}(t) \bar{K}_{2}(u),
$$

where $\bar{K}_{1}(t)$ and $\bar{K}_{2}(u)$ are the monosplines (2.10) and (2.11) with coefficients and knots (3.1); that is, formula (2.1) with coefficients and knots (3.1). The exact bound for the remainder of this formula is

$$
\bar{E}\left[V_{p}^{r, s}\right]=M\left\|\bar{K}_{1}(\cdot)\right\|_{q}\left\|\bar{K}_{2}(\cdot)\right\|_{q} .
$$

As the monosplines $\bar{K}_{1}(t)$ and $\bar{K}_{2}(u)$ define the asymptotically optimal quadrature formulas (3.1) with $l=m$ and $l=n$ on the sets $W_{p}^{r}$ and $W_{p}^{s}$, respectively, [3,pp. 22-26], we have by [3, pp. 108-109] the equalities

$$
\begin{gathered}
\left\|\bar{K}_{1}(\cdot)\right\|_{q}=\frac{B_{r, q}}{r ! m^{r}}\left(1+\frac{C_{1}}{m}\right)^{1 / q}, \quad\left\|\bar{K}_{2}(\cdot)\right\|_{q}=\frac{B_{s, q}}{s ! n^{s}}\left(1+\frac{C_{2}}{n}\right)^{1 / q}, \\
\lim _{m \rightarrow \infty} \frac{\left\|K_{1}^{*}(\cdot)\right\|_{q}}{\left\|\bar{K}_{1}(\cdot)\right\|_{q}}=\lim _{n \rightarrow \infty} \frac{\left\|K_{2}^{*}(\cdot)\right\|_{q}}{\left\|\bar{K}_{2}(\cdot)\right\|_{q}}=1 .
\end{gathered}
$$

Then by (3.3) and (3.4) the equality

$$
\lim _{m, n \rightarrow \infty} E^{*}\left[V_{p}^{r, s}\right] / \bar{E}\left[V_{p}^{r, s}\right]=1
$$

and the equality (3.2) hold.

The theorem is proved.

\section{COROllary. Consider the formula}

$$
\begin{aligned}
\iint_{D} f(x, y) d x d y= & \sum_{k=1}^{m} \sum_{j=1}^{n^{2}} \bar{A}_{k}^{(m)} \bar{B}_{j}^{\left(n^{2}\right)} f\left(\frac{k-1}{m-1}, \frac{j-1}{n^{2}-1}\right) \\
& +\sum_{k=1}^{m^{2}} \sum_{j=1}^{n} \bar{A}_{k}^{\left(m^{2}\right)} \bar{B}_{j}^{(n)} f\left(\frac{k-1}{m^{2}-1}, \frac{j-1}{n-1}\right) \\
& -\sum_{k=1}^{m} \sum_{j=1}^{n} \bar{A}_{k}^{(m)} \bar{B}_{j}^{(n)} f\left(\frac{k-1}{m-1}, \frac{j-1}{n-1}\right)+E_{1}(f) .
\end{aligned}
$$

Let $f(x, y) \in U_{p}^{r, s}$. Then

$$
\begin{aligned}
\left|E_{1}(f)\right| \leqslant & \frac{A N B_{r, q}}{r ! m^{2 r}}\left(1+\frac{C_{1}}{m}\right)^{1 / q}+\frac{B P B_{s, q}}{s ! n^{2 s}}\left(1+\frac{C_{2}}{n}\right)^{1 / q} \\
& +\frac{M B_{r, q} B_{s, q}}{r ! s ! m^{r} n^{s}}\left(1+\frac{C_{1}}{m}\right)^{1 / q}\left(1+\frac{C_{2}}{n}\right)^{1 / q}
\end{aligned}
$$

where $A$ and $B$ do not depend on $m$ and $n$,

$$
\left|\bar{A}_{1}^{(m)}\right|+\cdots+\left|\bar{A}_{m}^{(m)}\right| \leqslant A, \quad\left|\bar{B}_{1}^{(n)}\right|+\cdots+\left|\bar{B}_{n}^{(n)}\right| \leqslant B .
$$

The proof is the same as the proof of the Corollary following Theorem 2.1.

4. Comparison of the Formulas. Example. Consider the formula (1.5) with $l=m^{2}$, $\nu=n^{2}$ and the formulas obtained in the Corollaries of Sections 2 and 3. For simplicity we take $m=n, r=s$. As follows from [3] and Sections 1-3 the optimal 
formula (1.5) and the corresponding formulas (2.14), (2.17) and (3.5) have the same estimate $O\left(\mathrm{~m}^{-2 r}\right)$. However, formulas (2.14), (2.17) and (3.5) have a remarkable advantage over the optimal formula (1.5). The latter uses $m^{4}$ values of $f(x, y)$, while formulas (2.14), (2.17) and (3.5) use only $m^{2}(2 m+1)$ values of function $f(x, y)$.

Example. We compare the evaluation of the integral

$$
I=\iint_{D} \frac{1+(1-x-y+x y)^{2.5}}{0.5+\sin (x y)} d x d y=1.595958 \ldots
$$

by the optimal formula (1.5) with $l=\nu=m^{2}$ and by formula (2.14) with $m=n$. Both formulas are taken for the set $W_{\infty}^{2,2}$. By [3,pp. 50-51] we have

$$
\begin{aligned}
x_{k}^{(m)} & =y_{k}^{(m)}=(2 k-2+0.5 \sqrt{3}) h_{m}, \quad k=1, \ldots, m, \\
A_{1}^{(m)} & =A_{m}^{(m)}=B_{1}^{(m)}=B_{m}^{(m)}=(1+0.5 \sqrt{3}) h_{m}, \\
A_{k}^{(m)} & =B_{k}^{(m)}=2 h_{m}, \quad k=2, \ldots, m-1, \\
h_{m} & =(2 m+\sqrt{3}-2)^{-1} .
\end{aligned}
$$

Let $I_{m}$ be the approximate value of $I$ obtained by formula (2.14) with $q_{m}=2 m^{3}+m^{2}$ values of $f(x, y)$, and let $I_{m}^{\prime}$ be the approximate value of $I$ obtained by the optimal formula (1.5) with $k_{m}=m^{4}$ values of $f(x, y)$. We obtain for $m=3,6,9$

\begin{tabular}{crcrc}
\hline$I_{m}$ & $q_{m}$ & \multicolumn{1}{c}{$I_{m}^{\prime}$} & \multicolumn{1}{c}{$k_{m}$} & $m$ \\
\hline $1.59480 \ldots$ & 63 & $1.59484 \ldots$ & 81 & 3 \\
$1.595899 \ldots$ & 468 & $1.595901 \ldots$ & 1296 & 6 \\
$1.595947 \ldots$ & 1539 & $1.595947 \ldots$ & 6561 & 9 \\
\hline
\end{tabular}

The superiority of formula (2.14) over the optimal formula of the form (1.5) is obvious.

Acknowledgement. I am obliged to Professor L. Collatz and to Professor N. Dyn for stimulating discussions on the material presented in this paper.

School of Mathematical Sciences

Tel-Aviv University

Ramat-Aviv, Tel-Aviv, Israel

1. A. H. Stroud, Approximate Calculation of Multiple Integrals, Prentice-Hall, Englewood Cliffs, N. J., 1971.

2. S. Soвolev, Introduction to the Theory of Cubature Formulas, “Nauka”, Moscow, 1974. (Russian)

3. M. Levin \& J. Girshovich, Optimal Quadrature Formulas, Teubner Verlag, Leipzig, 1979.

4. S. M. Nikolsky, Quadrature Formulas, "Nauka", Moscow, 1980. (Russian)

5. W. J. GoRDON, "Distributive lattices and the approximation of multivariate functions," in Approximations with Special Emphasis of Spline Functions (I. J. Schoenberg, ed.), Academic Press, New York and London, 1969, pp. 223-277.

6. F. J. Delvos \& H. Posdorf, "N-th order blending," in Constructive Theory of Functions of Several Variables (W. Schempp and K. Zeller, eds.), Springer-Verlag, Berlin, Heidelberg, and New York, 1977, pp. 53-64.

7. M. Levin \& J. GirShovich, "Extremal problems for cubature formulas," Soviet Math. Dokl., v. 18, 1977, pp. $1355-1358$. 
8. A. SARD, “Best approximate integration formulas, best approximation formulas," Amer. J. Math., v. 71, 1949, pp. 80-91.

9. S. M. Nikolsky, "To the question of estimations of approximation with quadrature formulas," Uspekhy Mat. Nauk. v. 2 (36), 1950, pp. 165-177. (Russian)

10. I. J. SchónBerg, "Spline interpolation and best quadrature formulas," Amer. J. Math., v. 70, 1964, pp. 143-148.

11. M. Levin, "Extremal problem for one class of functions," Izv. Akad. Nauk ESSR, Fiz., Mat. $i$ Tekhn. Nauk, v. 12, 1963, pp. 141-145. (Russian)

12. A. ZenSYKBAEv, "On one property of best quadrature formulas," Mat. Zametki v. 23, 1978, pp. 551-562. (Russian)

13. V. I. KRYLOV, Approximate Calculation of Integrals, Macmillan, New York, 1962. 